\title{
Experimental study of residual displacements of double concave friction-pendulum isolation systems
}

\author{
Residual displacements of friction pendulum isolation systems
}

[Antonio Di Cesare, Felice Carlo Ponzo, Gianmarco Leccese, Domenico Nigro]

\begin{abstract}
After an earthquake, non-negligible residual displacements can occur if the isolation system does not possess a good restoring capability. In this paper the residual displacement of friction pendulum isolation systems is investigated through an extensive experimental campaign of controlled-displacement and seismic input experiments, both under uni-directional motion. A sequence of natural earthquakes was applied in three different lubrication conditions of sliding surfaces, corresponding to High, Medium and Low Friction. In order to improve current design method of sliding isolation systems, a simple equation for the estimation of the maximum expected residual displacements is proposed and compared with the experimental results.
\end{abstract}

Keywords - Seismic isolation, Double concave friction pendulum, Friction coefficient, Residual displacements, Shake table testing

\section{Introduction}

The underlying concept of base isolation is the uncoupling of horizontal building movement from ground motions using a flexible isolation layer made with either elastomeric bearings or sliding bearings. Typical flat sliding isolators use friction between composite materials, usually PTFE and stainless steel plates, as its energy dissipation system without any restoring capabilities.

The Friction Pendulum System (FPS) is one of the most widely techniques currently used for seismic isolation of buildings and bridges, with both energy dissipation and restoring capabilities, given by the pendulum action of the vertical load $W$, that generates the restoring force, the restoring stiffness $K_{p}$, and the friction force $F_{0}$. Based on the pendulum mechanism, the natural period $T$ of the isolated structure becomes independent by the mass of the superstructure $M$, as it only depends on the radius of the sliding surface $R$, as defined by (1).

$$
\begin{gathered}
K_{p}=\frac{W}{R}=\frac{M \cdot g}{R} \quad F_{0}=\mu \cdot W \\
T=2 \pi \sqrt{\frac{M}{K_{p}}}=2 \pi \sqrt{\frac{R}{g}}
\end{gathered}
$$

The force-displacement relationship of FPS consists in a bi-linear hysteresis loop (Fig. 1) defined by the restoring

Antonio Di Cesare, Felice Carlo Ponzo, Gianmarco Leccese, Domenico Nigro

School of Engineering, University of Basilicata Italy stiffness $K_{p}$, and lateral force $F=F_{0}+K_{p} \cdot d$, at the horizontal displacement $d$. The initial stiffness $K_{i}$ is referred to the pre-sliding stage of motion with a quasi-rigid behaviour.

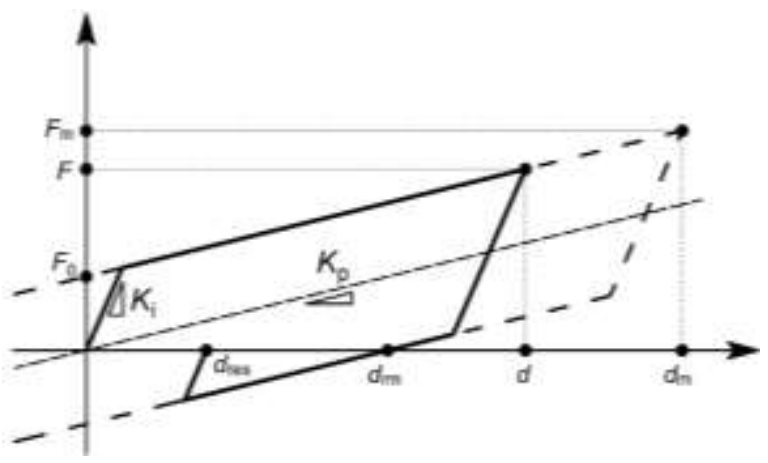

Figure 1. Idealized bilinear hysteretic behaviour

A remarkable improvement of the performance of the FPS isolator is obtained by introducing a second sliding surface. The resulting DCFP bearing consists of two steel concave plates (with radius $R_{I}$ and $R_{2}$ ) separated by a friction slider, which produces two independent pendulum response mechanisms and can be modeled as a combination in series of two FPSs. A DCFP bearing allows reaching greater displacements as compared to a corresponding FPS isolator with similar in plan dimensions, then the primary advantage of employing DCFP bearings is the cost savings that can be achieved through their more compact size. In the case of equal radius of curvature $\left(R_{l}=R_{2}\right)$ and same coefficient of friction $\mu$ on the upper and lower surfaces, sliding occurs simultaneously on both surfaces and the behaviour to resemble the functioning of the DCFP becomes equal to that of a FPS with equivalent radius $R_{e}=R_{1}+R_{2}$, and same friction coefficient [1].

In the ending stage of ground motion ("coda" stage), the presence of friction causes residual displacement when the frictional force is greater than restoring force of the curved surface. A curved surface bearing reaches the static equilibrium condition (Fig. 1) when $d_{r e s}<d_{r m}$, where $d_{r m}$ is the maximum static residual displacement defined by (2).

$$
d_{r m}=F_{0} / K_{p}=\mu \cdot R
$$

The value of $d_{r m}$ depends only on the characteristics of the isolation system and it represents the boundary condition of the residual displacement. The magnitude of the residual displacements depends on the restoring capability of the isolators.

In this paper, the extensive experimental campaign performed within the project JETBIS (Joint Experimental Testing on Base Isolation Systems) which involved different 
Italian universities has been described. The cyclic behaviour of Double Concave Friction Pendulum (DCFP) bearings is defined by means of controlled-displacement tests conducted at the University of Basilicata, considering 3 different friction conditions of the sliding surfaces of the bearings, namely High, Medium and Low friction. Several shaking table tests have been performed at the University of Naples "Federico II" applying a sequence of 19 seismic inputs, composed by 8 natural earthquakes at various intensities, and considering the same 3 different friction conditions. The experimental results are mainly discussed in terms of maximum and residual displacements.

Based on the experimental results, an estimation of the maximum residual displacement dependent only on the characteristics of the isolation system is proposed in the paper. This simplified design tool is valid for all considered friction coefficients and all selected ground motions, even when initial slider offsets are present. Studies confirm that a sufficient restoring capability is guaranteed when the ratio $\mathrm{d}_{\max } / \mathrm{d}_{\mathrm{rm}}>0.5$.

\section{Experimental investigations}

\section{A. Specimen description}

The model structure used in the shaking table testing consists of a three dimensional one-storey steel frame, designed as inverted pendulum structure.
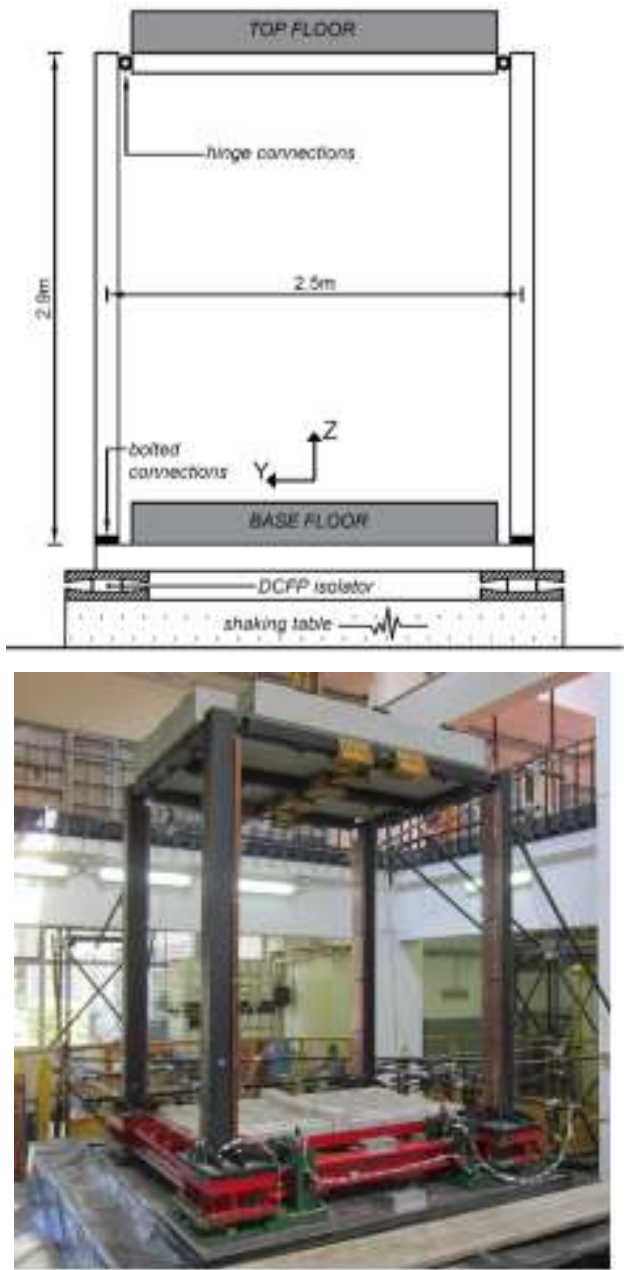

Figure 2. Views of the experimental model on the shaking table.
The storey height is $2.9 \mathrm{~m}$ and the rectangular plan is single bay in both directions with dimensions of $2.5 \times 2.0 \mathrm{~m}$. The base rigid floor, needed for seismic isolation, consists of a horizontal braced steel frame with HEM160 section, for external primary beams, and UPN80 section, for internal secondary beams. At top floor, all the beams are S275 steel rolled square hollow section $(120 \times 120 \times 12.5 \mathrm{~mm})$. The four columns are of $\mathrm{C} 45$ steel welded square hollow section ( $150 \times 150 \times 15 \mathrm{~mm})$ with bolted beam-column connections, at the top floor, and fixed connections at the base floor. Additional masses were added placing a $250 \mathrm{~mm}$ thick concrete slab, on the top floor, and 40 concrete blocks (23.8 $\mathrm{kg}$ each), on the base floor. The total weight of the model (frame, base and added masses) on top of the isolation level is $80.45 \mathrm{kN}$ (49.05 kN at base and $31.40 \mathrm{kN}$ at top floor). Fig. 2 shows an overview of the experimental model on the shaking table and a scheme of testing setup. Uni-directional seismic loads were applied parallel to the long side of structure (Y-axis direction).

In this study, the isolated model was considered as 1:3 scaled structure, in order to reduce the maximum displacement coherently with the limits of the test facility. Four DCFP isolators accommodating for horizontal displacements and rotations about the vertical axes were placed below the columns, under the base rigid floor. The vertical load is equally distributed on each isolator with an approximate value of $20.11 \mathrm{kN}$. Each bearing consists of two identical concave sliding surfaces (same radius of curvature and friction coefficient) and a non-articulated rigid slider. The equivalent radius of curvature is $R_{e}=1805 \mathrm{~mm}$, approximately twice geometric radius of curvature of the sliding surfaces $\left(R_{1}=R_{2}=925 \mathrm{~mm}\right)$, modified for the height of the slider. The overall horizontal displacement capacity $d_{m}$ is $\pm 180 \mathrm{~mm}$. The isolation system is designed coherently with the scale adopted for the superstructure [2]. The sliding material, at steel plates-slider interface, consists of an UltraHigh Molecular Weight Poly-Ethylene (UHMW-PE) high bearing capacity composite material, produced by FIP Industriale [3]. The seismic response of the structure was recorded by 16 servo-accelerometers and 14 displacement transducers, placed both on the top and at the base level, for three-directional acquisitions of accelerations and displacements.

\section{B. Characterization of DCFP bearings}

During the entire design life of the bearings the friction condition can vary due to the effects of aging and/or contamination, then the properties of sliding isolators can change and modify the seismic response of the isolated structure.

In this study, 3 different conditions of the lubrication of surfaces have been considered for the DCFP bearings to modify the values of the friction coefficient $\mu$, as follow sequence:

1. High Friction, surfaces without lubrication;

2. Low Friction, surfaces with a silicone based (lithium soap) lubricant applied to the top and bottom face of the rigid slider;

3. Medium Friction, surfaces cleaned up by lubrication. 
Characterization testing to determinate frictional properties of the DCFP bearings has been performed at structural laboratory of University of Basilicata (Potenza, Italy). The testing machine is capable of testing bearings with controlled-displacements under constant vertical load tests. A load cells mounted directly beneath the actuators measures reaction forces and lateral displacement is measured by an internally mounted LVDT on the horizontal actuator. The four bearings were tested all together under a total vertical compressive load of $128 \mathrm{kN}$, the minimum value available by test machine, corresponding to $32 \mathrm{kN}$ for each isolator. A series of sinusoidal lateral displacement were imposed with slow sliding velocity $(v \approx 50 \mathrm{~mm} / \mathrm{s})$ and fast sliding velocity $(v \approx 400 \mathrm{~mm} / \mathrm{s})$ in accordance to the testing procedure adopted by EN:15129 [4].

The friction coefficients obtained are listed in Table I, for each sliding surfaces condition and test velocity. Fig. 3 shows experimental force-displacement relationships of the displacement cycles correspondent to the capacity of the DCFP bearings $\left(d_{m}= \pm 180 \mathrm{~mm}\right)$.

Due to the low velocity values reached during the coda stage of earthquakes, in the following of the paper, the friction coefficient is only referred to the value obtained during slow sliding velocity test $\left(\mu=\mu_{\text {slow }}\right)$

TABLE I. MAIN PARAMETERS OF DCFP BASED ON THE TESTS.

\begin{tabular}{|c|c|c|c|c|}
\hline $\begin{array}{c}\text { Friction } \\
\text { condition }\end{array}$ & Surfaces & $\begin{array}{c}\boldsymbol{\mu}_{\text {slow }} \\
{[\mathbf{\%}]}\end{array}$ & $\begin{array}{c}\boldsymbol{\mu}_{\text {fast }} \\
{[\mathbf{\%}]}\end{array}$ & $\begin{array}{c}\mathbf{d}_{\text {rm }} \\
{[\mathbf{m m}]}\end{array}$ \\
\hline High & Non-lubricated & 12.0 & 20.0 & 216 \\
\hline Medium & Cleaned up & 5.0 & 8.0 & 90 \\
\hline Low & Lubricated & 2.5 & 5.0 & 45 \\
\hline
\end{tabular}
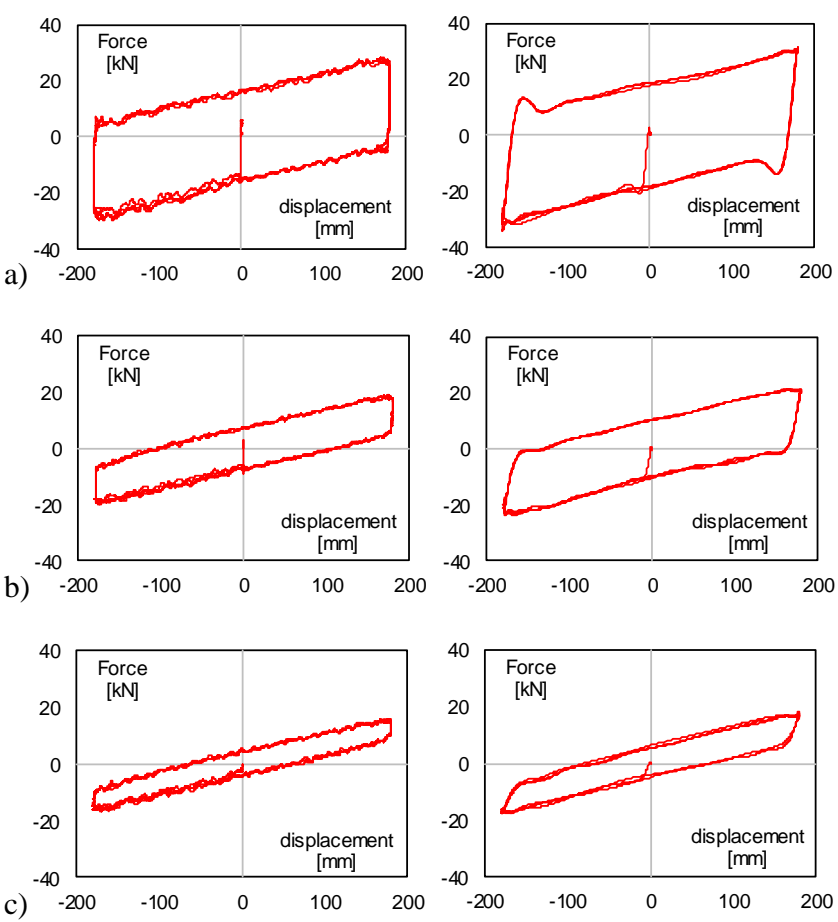

Figure 3. Experimental cyclic behaviour of DCFP bearings at slow (left) and fast (right) velocity: a) High friction; b) Medium Friction and c) Low Friction.

\section{Shake table testing}

\section{A. Seismic Inputs}

A set of seven natural earthquakes was selected from the European strong-motion database. According with the Italian Seismic Code [5], the considered horizontal waveforms are in compliance with the collapse prevention Limit State for a strategic structure (functional class IV) located in Naples (Italy), founded on rigid soil (type A) with a nominal life of 100 years (which corresponds to a 1898year return period). An additional waveform, compatible with medium soil (type B), was also applied. In Table II the main characteristics of the considered recording are summarized. To ensure consistency with the scale of the experimental model, the recordings were scaled down in time by a factor of $1 / \sqrt{ } 3$.

TABLE II. SEISMIC INPUTS SELECTED FOR EXPERIMENTAL TESTING.

\begin{tabular}{|c|c|c|c|c|c|}
\hline $\begin{array}{c}\text { Waveform } \\
\text { ID }\end{array}$ & Seismic event & Date & Mw & $\begin{array}{c}\text { PGAref } \\
{\left[\mathbf{a}_{\mathrm{g}} / \mathbf{g}\right]}\end{array}$ & Soil \\
\hline 7142 & Bingol & $01 / 05 / 2003$ & 6.3 & 0.25 & $\mathrm{~A}$ \\
\hline 55 & Friuli & $06 / 05 / 1976$ & 6.5 & 0.25 & $\mathrm{~A}$ \\
\hline 200 & Montenegro & $15 / 04 / 1979$ & 6.9 & 0.25 & $\mathrm{~A}$ \\
\hline 428 & Etolia & $18 / 05 / 1988$ & 5.3 & 0.25 & $\mathrm{~A}$ \\
\hline 372 & Lazio-Abruzzo & $07 / 05 / 1984$ & 5.9 & 0.25 & $\mathrm{~A}$ \\
\hline 290 & Campano Lucano & $23 / 11 / 1980$ & 6.9 & 0.25 & $\mathrm{~A}$ \\
\hline 287 & Campano Lucano & $23 / 11 / 1980$ & 6.9 & 0.25 & $\mathrm{~A}$ \\
\hline 196 & Montenegro & $15 / 04 / 1979$ & 6.9 & 0.45 & $\mathrm{~B}$ \\
\hline
\end{tabular}

The seismic sequence is composed by 19 seismic inputs applied by increasing the PGA intensities. In the first part of sequence, the 287 earthquake was applied at lower intensities equal to $25 \%, 50 \% 75 \%$ and $100 \%$ of $\mathrm{PGA}_{\text {ref }}$. Then, in the second part, all the considered earthquakes

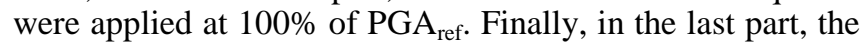
287-7142-55-196 earthquakes were applied at higher intensities corresponding to $150 \%$ and $200 \%$ of PGA $_{\text {ref }}$.

The same sequence was applied for the considered friction conditions (High - Medium - Low). The application verse of the seismic acceleration is the same for all sequences. At the end of each sequence the bearings were globally recentered. The complete input acceleration sequence is reported in Fig. $4 \mathrm{a}$.

\section{B. Experimental results}

In this paper the experimental are described in terms of displacement of the seismic isolation system observed for all shaking table tests. Fig. 4b, 4c, 4d show the experimental base displacements as recorded during the earthquake sequences for each friction condition.

From the first and third part of seismic sequence, it is possible to observe that $d_{\max }$ increases linearly with the PGA level increasing. In the second part of seismic sequence, when the same PGA intensities were applied, it is clear that $d_{\max }$ is extremely variable and depends mainly on the characteristics of the ground motion rather than the reference seismic intensity $\left(\mathrm{PGA}_{\text {ref }}\right)$. 

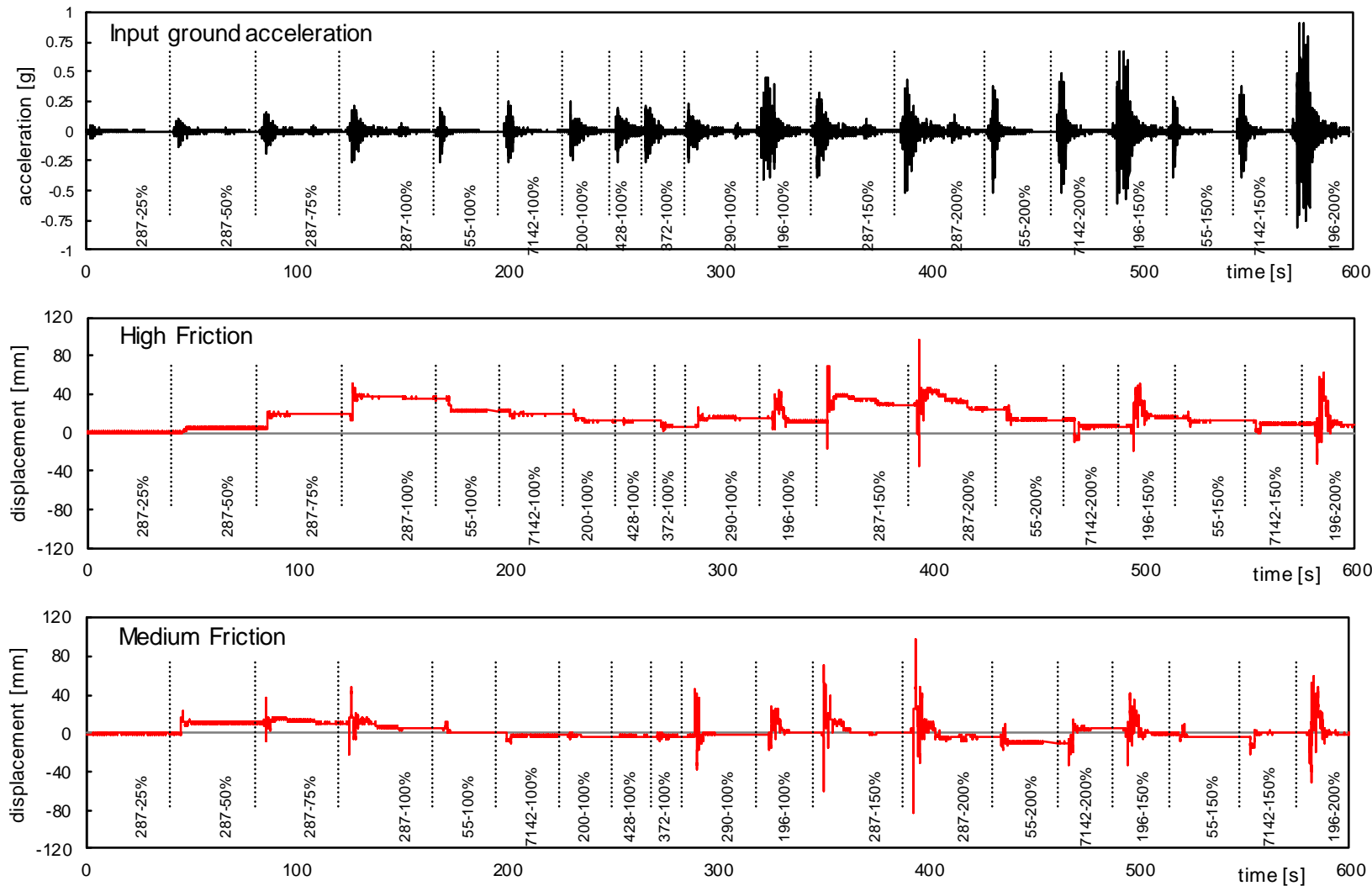

d)

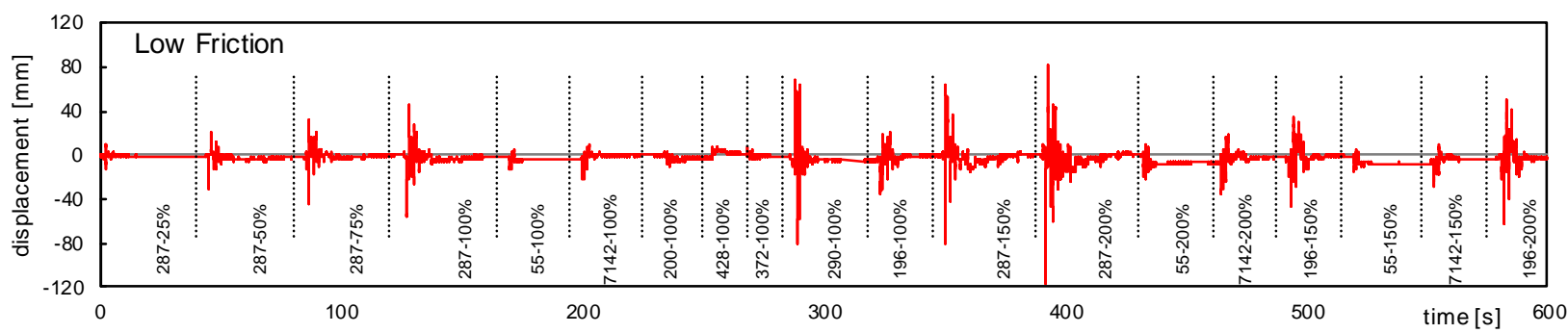

Figure 4. a) Seismic input sequence; b); c) and d) base displacement histories recorded in all friction conditions

Fig. $4 \mathrm{~b}$ shows that for High Friction condition the highest values of residual displacements $d_{\text {res }}$ have been recorded, due to the lower restoring capability. In the Medium and Low Friction conditions the $d_{\text {res }}$ values are negligible. The variation of the results within the same friction condition confirms that the value of $d_{\text {res }}$ is strictly connected to the characteristics of the coda stage of the seismic input, as well as the DCFP bearings restoring capability.

Fig. 5a shows the $d_{m a x} / d_{r m}$ ratio obtained in all seismic tests performed. The reference value of the ratio $d_{m a x} / d_{r m}=$ 0.5 , proposed by [6] for a good restoring capability, is also reported in the graph.

Fig. $5 \mathrm{~b}$ shows the ratio $d_{r e s} / d_{r m}$. It is possible to observe that maximum values of the $d_{\text {res }} / d_{r m}$ ratio are mainly in the range between $0.05 \div 0.10$ and occasionally exceed the value of 0.15 . Results are in line with previous studies, which have shown that residual displacement values are approximately an order of magnitude less than the maximum static residual displacement $\mathrm{d}_{\mathrm{rm}}[1]$. a)

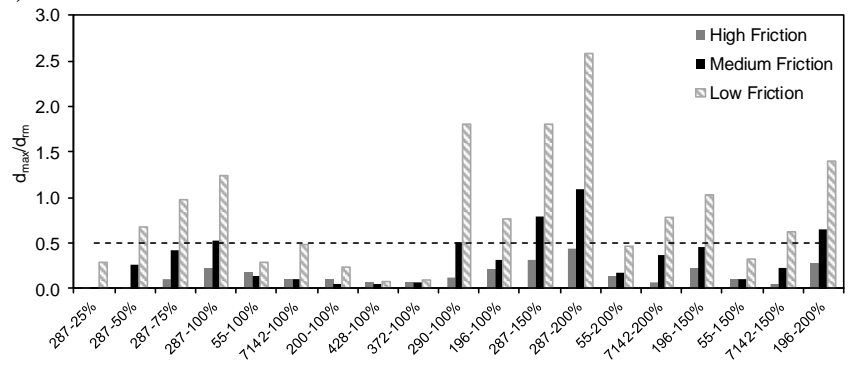

b)

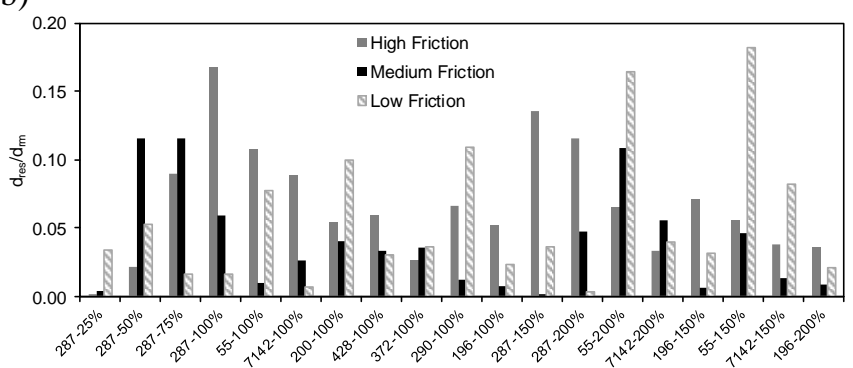

Figure 5. Experimental results in all friction conditions: a) $d_{\max } / d_{\mathrm{rm}}$ ratio; b) $d_{\text {res }} / d_{\text {rm }}$ ratio 


\section{Estimation of maximum residual displacements}

Fig. 6 shows the normalized residual displacements $d_{\text {res }} / d_{\max }$ and $d_{\text {res }} / d_{r m}$ obtained from all shaking table tests as function of the ratio $d_{\max } / d_{r m}$. Experimental results confirm that the isolation system exhibits a good restoring capability when the ratio $d_{\max } / d_{r m}>0.5$. In any case, the residual displacements are less than $10 \%$ of the corresponding maximum displacements when the ratio $d_{\max } / d_{r m}>0.7$.

On the base of the experimental results, a simplified relation for the estimation of the maximum residual displacement $d_{\text {res }}$ as function only of the characteristics of the isolators is proposed. The estimation tool (3) provides with sufficient accuracy a maximum value of $d_{r e s}$, including over the $90 \%$ of the experimental data.

$$
d_{r e s, 90 \%}=0.12 \cdot d_{r m}=0.12 \cdot \mu \cdot \mathrm{R}
$$

In Fig. 6 the envelope curve obtained through the relation (3) is also shown. The proposed equation allows to easily estimate the maximum residual displacement with good safety level for all values of the ratio $d_{\max } / d_{r m}$.
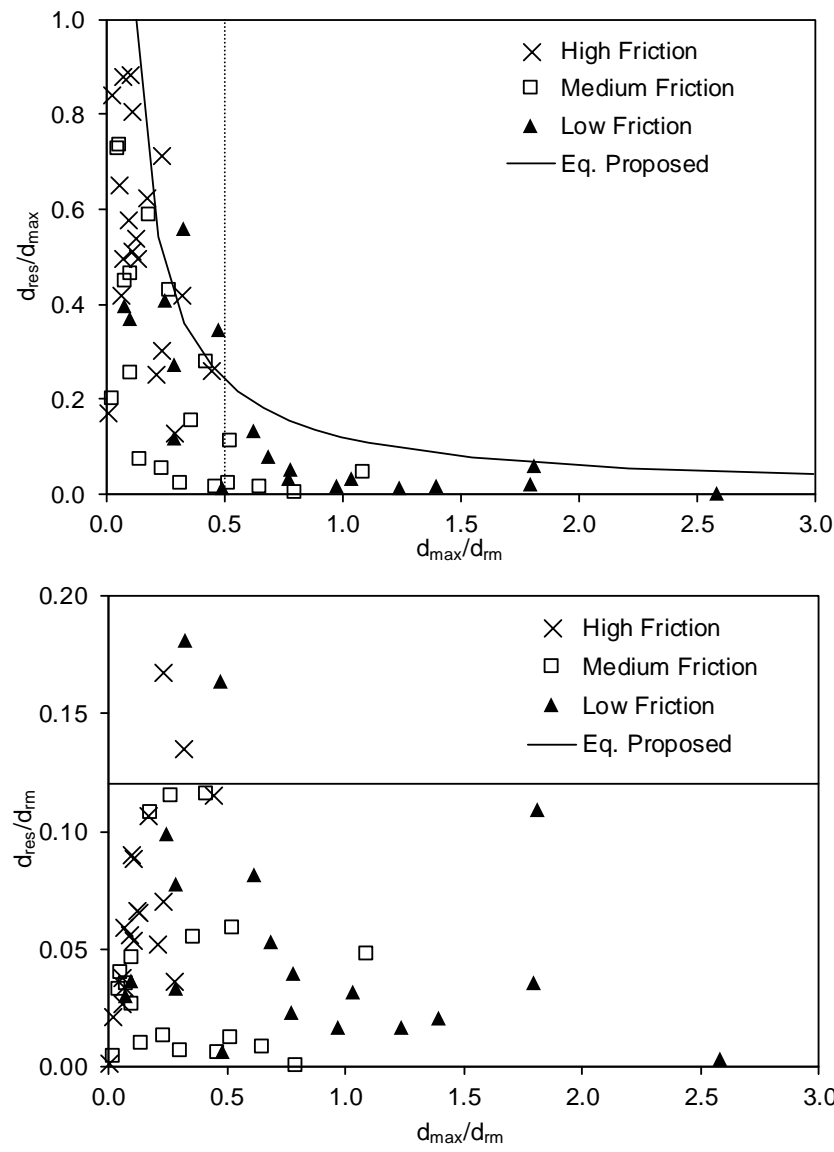

Figure 6. Normalized residual displacements a) $d_{\text {res }} / d_{\max }$ and b) $d_{r e s} / d_{r m}$, both function of the ratio $d_{\max } / d_{r}$

\section{Conclusions}

In this paper, the restoring capability of isolation systems based on Double Concave Friction Pendulum (DCFP) bearings is investigated through an extensive experimental campaign. Coherently with previous studies, the ratio between maximum displacement and maximum static residual displacement $d_{\max } / d_{r m}$ appears to be the main parameters affecting the restoring capability of the isolation system. The overview of the experimental results confirms that the maximum displacement $d_{\max }$ is mainly affected by the characteristics of the ground motion as well as the PGA intensity. In all considered friction conditions, the initial offset does not influence the maximum displacement when stronger earthquakes occur because during the strongmotion stage, the initial offset tends to be eliminated. On the contrary, in the case of High friction conditions when weaker earthquakes, the initial bearing offset could increase the maximum displacement of the bearings. From experimental tests, the ratio $d_{r e s} / d_{\max }$ is less than $10 \%$ when the ratio $d_{\max } / d_{r m}>0.7$, also when initial offsets are relevant.

Based on the experimental results, in this paper a proposal for the estimation of maximum residual displacement has been presented as function only of the characteristics of the isolation system. The simple design tool defines a maximum value of residual displacement $d_{\text {res }}$ equal to $12 \%$ of the maximum static residual displacement $d_{r m}$ of the isolation system, independently from the characteristics of the earthquakes sequence. This value represents with sufficient accuracy, over the $90 \%$ of the experimental data.

\section{Acknowledgment}

The present work was funded by ReLUIS (Italian network of university laboratories of earthquake engineering) Consortium within the ReLUIS/DPC 20142018 research program

\section{References}

[1] Fenz DM \& Constantinou MC (2006). Behaviour of the double concave friction pendulum bearing. Earthquake engineering \& structural dynamics, 35(11), 1403-1424.

[2] Ponzo FC, Di Cesare A, Nigro D, Simonetti M, Leccese G (2014). Shaking table tests of a base isolated structure with double concave friction pendulums. Bulletin of the New Zealand Society for Earthquake Engineering, 48(2), 136-144.

[3] FIP Industriale (2013). Catalogue S04 - Curved Surface Sliders, Padova, Italy.

[4] CEN (2009). Anti-seismic devices. European Committee for Standardization, EN 15129:2009. Bruxelles, Belgium.

[5] NTC (2008). Norme tecniche per le costruzioni. D.M. 14 Gennaio 2008.

[6] Katsaras CP, Panagiotakos TB, Kolias B (2008). Restoring capability of bilinear hysteretic seismic isolation systems. Earthquake Engineering \& Structural Dynamics, 37(4), 557-575. doi:10.1002/eqe.772 